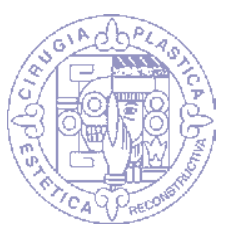

\title{
Depresión y cirugía plástica
}

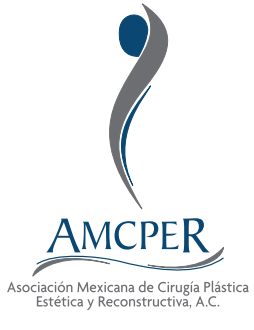

\author{
Depression and plastic surgery
}

\section{Dr. André Víctor Baldin,* Dr. José E Telich-Tarriba,* Dra. Jimena Díaz-Lozano Dovalí,** Dr. Armando Apellaniz Campo,*** Dra. Leticia Sánchez Méndez****}

Palabras clave: Depresión, cirugía plástica, trastornos depresivos.

Key words: Depression, plastic surgery, depressive disorders.

* Residente de

Cirugía Plástica y Reconstructiva. Hospital General «Dr. Manuel Gea González». ** Psicóloga Clínica. Universidad Iberoamericana. *** Jefe de la Clínica de Plástica General y Reconstrucción Auricular. Hospital General «Dr. Manuel Gea González».

**** Psicóloga

Clínica del Servicio de Cirugía Plástica y Reconstructiva. Hospital General «Dr. Manuel Gea González».

Los autores de este artículo no tienen conflicto de intereses qué declarar.

Recibido:

16 noviembre 2017 Aceptado para publicar: 05 diciembre 2017

\section{RESUMEN}

La autoimagen corporal es uno de los constructores de la autoestima y la insatisfacción con la misma es uno de los principales motivos para la búsqueda de procedimientos estéticos. La cirugía plástica es un componente esencial para la recuperación psicológica del paciente. De hecho, hasta un $48 \%$ de los pacientes que buscan atención por un cirujano plástico padecen de algún tipo de trastorno psicológico. Es por este motivo que la identificación de los pacientes con rasgos de trastornos depresivos se vuelve fundamental. Este artículo tiene por objetivo realizar una revisión sistemática de la literatura referente a la depresión y cirugía plástica, así como educar y orientar a los cirujanos plásticos acerca de esta asociación, que en muchas ocasiones puede ser catastrófica.

\section{ABSTRACT}

Body self-image is one of the builders of self-esteem, dissatisfaction with it is one of the main reasons for the search for aesthetic procedures, and plastic surgery is an essential component for the restoration of the psychological health of the patient. In fact, up to $48 \%$ of patients seeking care by a plastic surgeon suffer from some type of psychological disorder. It is for this reason that the identification of patients with features of depressive disorders becomes fundamental. The objective of this article is to carry out a systematic review of the literature regarding depression and plastic surgery, as well as to educate and guide plastic surgeons about this association, which in many cases can be catastrophic.

\section{INTRODUCCIÓN}

$\mathrm{L}$ a autoimagen corporal es uno de los componentes de la autoestima, misma que está formada por una variedad de factores, como antecedentes históricos, culturales, sociales, biológicos, religiosos e individuales de cada paciente. ${ }^{1}$

La insatisfacción con la autoimagen corporal es el principal factor para la búsqueda de procedimientos estéticos, y la cirugía plástica es un componente esencial para la restauración del aspecto psicológico del paciente, mejorar la integración con su entorno biopsicosocial y proporcionar bienestar y autoconfianza. ${ }^{1,2}$

Existen estudios que demuestran que hasta un $48 \%$ de pacientes que solicitan atención por un cirujano plástico padecen algún tipo de trastorno psicológico, sin importar el grado de afectación, y buscan en la cirugía plástica una oportunidad para lograr su satisfacción personal. El trastorno que se observa con más frecuencia es la depresión. ${ }^{3}$

\section{Revisión sistemática de la literatura}

Se realizó una búsqueda en PubMed y EBSCO de los últimos 15 años, utilizando las palabras clave: «cirugía plástica», «depresión», «trastornos depresivos» y «síntomas depresivos», en español e inglés.

De acuerdo con la Organización Mundial de la Salud (OMS), la depresión es una enfermedad que afecta a más de 300 millones de personas en el mundo. Puede iniciar a cualquier edad, aunque su mayor prevalencia se presenta entre los 15 y 45 años, e impacta en forma importante en la educación, productividad, funcionamiento y relaciones interpersonales. Se presenta como un conjunto de síntomas de predominio afectivo, tales como tristeza patológica, apatía, anhedonia, desesperanza, decaimiento, irritabilidad, sensación 
subjetiva de malestar e impotencia frente a las exigencias de la vida, aunque en mayor o menor grado también están presentes síntomas de tipo cognitivo, volitivo y somático, por lo que se puede hablar de una afectación global psíquica y física. ${ }^{4,5}$

En México se calcula que la tasa de prevalencia anual de depresión en la población adulta es del $4.8 \%$. Entre los jóvenes mexicanos, la encuesta mexicana de salud mental identificó que el $7.2 \%$ entre los 12 y 17 años sufre de trastorno depresivo, la mitad de los casos (54.7\%), calificados como graves. ${ }^{6,7}$

Además, la asociación entre trastornos depresivos y suicidio es alta: 30 veces mayor de la población general. Según la OMS, es posible establecer el diagnóstico de depresión hasta en el 35\% de los pacientes con intento de suicidio. ${ }^{7-9}$

\section{Etiología}

La depresión es todo un proceso multifactorial y complejo, interactúan factores propios del paciente, psicológicos y de conflicto interno, así como de alteración bioquímica a nivel del sistema nervioso central. Se desconoce el peso real de cada uno de ellos en relación con las circunstancias y momento de la vida en el que se desarrolla el trastorno depresivo. ${ }^{9,10}$

Además, existen variables que aumentan el riesgo de desarrollar depresión, que se pueden clasificar en factores personales, sociales, cognitivos, familiares y genéticos; sin embargo, a pesar de todos los esfuerzos para su estudio, la fisiopatología de la depresión todavía no está establecida al $100 \%{ }^{11}$

\section{DIAGNÓSTICO}

Se trata de una entidad clínica clasificada en los manuales diagnósticos CID-10 y DSM-V. Su diagnóstico lo debe realizar un médico especialista en psiquiatría o psicólogo, quienes lo hacen durante un interrogatorio dirigido y examen clínico minucioso. Sin embargo, los cirujanos plásticos también están expuestos a esos pacientes, motivo por el cual deben estar atentos para identificar estigmas de dicha enfermedad. ${ }^{4,5}$

El paciente con depresión puede presentar una serie de signos y síntomas, muchas veces inespecíficos, dificultando así su diagnóstico. A estos signos y síntomas los podemos dividir en dos grandes grupos: psíquicos y orgánicos: ${ }^{1,2,5}$

- Psíquicos: astenia, adinamia, tristeza, desánimo, apatía, falta de interés en su cuidado personal, inseguridad, negatividad, desesperanza, sentimiento de culpa, falta de concentración, irritabilidad, Ilanto fácil, impotencia e ideas suicidas.

- Orgánicos: insomnio o hipersomnia, alteraciones del apetito, pérdida de la libido, fatiga, astenia, adinamia, lentitud psicomotriz, disminución de la concentración, disminución del aprendizaje.

Es muy importante para los cirujanos plásticos elaborar una historia clínica completa e intentar identificar la naturaleza de los síntomas, uso o abuso de sustancias y antecedentes de depresión personal y familiar. ${ }^{12}$

Se han desarrollado muchas escalas y cuestionarios con el propósito de ayudar a la identificación y diagnóstico de la depresión. Beck y colaboradores desarrollaron una prueba/ cuestionario, conocido como Beck Depression Inventory (BDI), que ha demostrado ser un instrumento consistente y confiable, además de haber sido traducido, estandarizado y validado para su aplicación en México. Se trata de un cuestionario sencillo de aplicar y permite clasificar la severidad de los síntomas de depresión en mínimo, leve, moderado y grave. ${ }^{13-15}$

\section{Depresión y cirugía plástica}

La depresión se ha transformado en uno de los grandes desafíos de la medicina moderna, debido a su alta y creciente prevalencia y está considerada por muchos como la enfermedad del siglo XXI. ${ }^{16}$

Estudios recientes que correlacionan la depresión y la cirugía plástica demuestran una alta prevalencia, observándola hasta en el 32\% de la población. Además, reportan que el 20\% de los pacientes que buscan un procedimiento estético estuvieron acompañados en algún momento por un especialista en salud mental y el $18 \%$ utiliza algún tipo de medicamento antidepresivo. Algunos factores asociados a depresión y cirugía plástica son: tabaquismo, 
consumo excesivo de alcohol, uso de drogas y pacientes divorciadas. ${ }^{12,17,18}$

Existen dos vertientes cuando se habla de depresión y cirugía plástica. En la primera, los autores mencionan que la cirugía plástica se limita únicamente al plano físico, generando poca o incluso ninguna mejoría psíquica; sin embargo, un buen número de publicaciones ha demostrado que la cirugía plástica proporciona bienestar físico y psicológico, además de mejorar el grado de satisfacción personal, mejor grado de satisfacción de autoimagen, calidad de vida, autoestima, mejor interacción social y sexual, conjuntamente con la disminución de los síntomas depresivos. ${ }^{12,19-21}$

Es muy importante no confundir los beneficios de la realización de un procedimiento de cirugía plástica en pacientes con depresión, con la cirugía plástica psicológicamente segura. Las complicaciones psicológicas inherentes a procedimientos quirúrgicos pueden presentar tasas iguales, o incluso mayores a las de las complicaciones físicas. Pacientes que tienen complicaciones postoperatorias aumentan su riesgo de desarrollar en forma simultánea complicaciones psicológicas. Incluso pacientes que lucen un adecuado resultado estético pueden evolucionar en forma tórpida en el ámbito psicológico, muchas veces asociados con alteraciones de percepción de autoimagen y/o desequilibrio psicoemocional, no detectados muchas veces por los cirujanos plásticos. 1,3,12,22

Pacientes identificados con estigmas de depresión preoperatoria tienen hasta cinco veces más insatisfacción estética después del procedimiento quirúrgico en comparación con la población general. Dicha insatisfacción se puede presentar en grado variable, desde reclamos y quejas hasta síntomas depresivos mayores, incluso suicidio. Casos extremos pueden poner en peligro la integridad moral y física del cirujano, como persecución, difamación, amenazas, violencia física y verbal, además de demandas legales. ${ }^{12,19,23,24}$

Se han detectado características personales con un mayor número de síntomas depresivos en pacientes candidatas a mamoplastia de aumento. En este grupo de pacientes se identifica con mayor prevalencia antecedentes de enfermedades psicológicas/psiquiátricas, uso de psicofármacos (antidepresivos o inductores de sueño), disturbios de conducta y trastornos alimentarios. ${ }^{21,22,25-27}$

La clave para obtener buenos resultados en cirugía plástica es la selección del paciente. Algunos autores recomiendan el uso de una evaluación especializada por un psicólogo o psiquiatra de forma rutinaria en todos los procedimientos estéticos; sin embargo, se trata de un tema controvertido. Incluso el envío de un paciente sano a valoración psicológica puede causar el deterioro de la relación médico-paciente. 3,5,28,29

Concierne al cirujano plástico identificar que los pacientes que buscan procedimientos de cirugía plástica tengan una adecuada estabilidad psicológica y cuáles necesitarán una valoración especializada en salud mental. ${ }^{10,11,30-32}$

La identificación de estos pacientes es fundamental en la prevención de las complicaciones postoperatorias. Para ello, es esencial elaborar una historia clínica y examen clínico minuciosos, pudiendo incluso aplicar cuestionarios para depresión, como el BDI, que ayudará al cirujano a identificar trastornos depresivos y canalizarlos a un profesional especializado en salud mental. $3,5,33,34$

Pacientes en los que es recomendable realizar una evaluación psicológica inicial son los que tienen un grado elevado de exigencia con expectativas irreales de los resultados postoperatorios, pacientes insatisfechos con procedimientos quirúrgicos previos con adecuados resultados, pacientes con baja autoestima, historia de depresión, uso de antidepresivos, o que no saben el motivo real por el cual pretenden operarse o por motivos relacionados con problemas conyugales. $5,10,11$

\section{CONCLUSIONES}

Todavía no está establecido con certeza el impacto de los procedimientos de cirugía plástica en el comportamiento psicológico de los pacientes con depresión. Por ese motivo, la identificación de los pacientes con factores de riesgo predisponentes a conductas depresivas y su envío a un especialista en salud mental es fundamental. Dicho especialista emitirá un dictamen en el que autorizará o no el procedimiento quirúrgico y el mejor momento para hacerlo, o si necesitará tratamiento específico para su trastorno depresivo. 
El referir un paciente a valoración especializada en los casos indicados no hace ver temeroso al cirujano plástico; al contrario, demuestra madurez profesional $y$, sobre todo, preocupación con su paciente, reconociendo que se trata de un ser biopsicosocial.

\section{REFERENCIAS}

1. Slade PD. What is body image? Behav Res Ther. 1994; 32 (5): 497-502.

2. Sarwer DB, LaRossa D, Bartlett SP, Low DW, Bucky LP, Whitaker LA. Body image concerns of breast augmentation patients. Plast Reconstr Surg. 2003; 112 (1): 83-90.

3. Shridharani SM, Magarakis M, Manson PN, Rodriguez ED. Psychology of plastic and reconstructive surgery: a systematic clinical review. Plast Reconstr Surg. 2010; 126 (6): 2243-2251.

4. Bhugra D, Mastrogianni A. Globalisation and mental disorders. Overview with relation to depression. $\mathrm{Br} J$ Psychiatry. 2004; 184: 10-20.

5. American Psychiatric Association. DSM-V diagnostic and statistical manual of mental disorders. 5th ed. 2014 [Acceso 08 de abril de 2016]. Disponible en: http:// www.dsm5.org/about/Pages/faq.aspx

6. Wagner FA, González-Forteza C, Sánchez-García S, García-Peña C, Gallo JJ. Enfocando la depresión como problema de salud pública en México. Salud Ment. 2012; 35 (1): 3-11.

7. Sher L, Oquendo MA, Mann JJ. Risk of suicide in mood disorders. Clin Neurosci Res. 2001; 1 (5): 337-344.

8. Bertolote JM, Fleischmann A. Suicide and psychiatric diagnosis: a worldwide perspective. World Psychiatry. 2002; 1 (3): 181-185.

9. Gibbons RD, Hur K, Bhaumik DK, Mann JJ. The relationship between antidepressant medication use and rate of suicide. Arch Gen Psychiatry. 2005; 62 (2): 165-172.

10. Honigman RJ, Phillips KA, Castle DJ. A review of psychosocial outcomes for patients seeking cosmetic surgery. Plast Reconstr Surg. 2004; 113 (4): 1229-1237.

11. Sarwer DB, Gibbons LM, Magee L, Baker JL, Casas LA, Glat PM et al. A prospective, multi-site investigation of patient satisfaction and psychosocial status following cosmetic surgery. Aesthet Surg J. 2005; 25 (3): 263269.

12. Alagöz MS, Başterzi AD, Uysal AC, Tüzer V, Unlü $\mathrm{RE}$, Sensöz $\mathrm{O}$ et al. The psychiatric view of patients of aesthetic surgery: self-esteem, body image, and eating attitude. Aesthetic Plast Surg. 2003; 27 (5): 345-348.

13. Naraghi M, Atari M. A comparison of depression scores between aesthetic and functional rhinoplasty patients. Asian J Psychiatr. 2015; 14: 28-30.

14. Beck AT, Ward CH, Mendelson M, Mock J, Erbaugh J. An inventory for measuring depression. Arch Gen Psychiatry. 1961; 4: 561-571.

15. Jurado S, Villegas ME, Méndez L, Rodríguez F, Loperena V, Varela R. La estandarización del Inventario de Depresión de Beck para residentes de la Ciudad de México. Salud Mental. 1998; 21 (3): 26-31.

16. World Health Organization. Depression. Geneva: World Health Organization; 2012. Disponible en: http://www. who.int/topics/depression/

17. Zojaji R, Arshadi HR, Keshavarz M, MazloumFarsibaf M, Golzari F, Khorashadizadeh M. Personality characteristics of patients seeking cosmetic rhinoplasty. Aesthetic Plast Surg. 2014; 38 (6): 1090-1093.

18. Vargel S, Uluşahin A. Psychopathology and body image in cosmetic surgery patients. Aesthetic Plast Surg. 2001; 25 (6): 474-478.

19. Chahraoui K, Danino A, Frachebois C, Clerc AS, Malka G. Aesthetic surgery and quality of life before and four months postoperatively. Ann Chir Plast Esthet. 2006; 51 (3): 207-210.

20. Klassen AF, Pusic AL, Scott A, Klok J, Cano SJ. Satisfaction and quality of life in women who undergo breast surgery: a qualitative study. BMC Womens Health. 2009; 9: 11

21. von Soest T, Kvalem IL, Roald HE, Skolleborg KC. The effects of cosmetic surgery on body image, self-esteem, and psychological problems. J Plast Reconstr Aesthet Surg. 2009; 62 (10): 1238-1244.

22. Joiner TE Jr. Does breast augmentation confer risk of or protection from suicide? Aesthet Surg J. 2003; 23 (5): 370-375.

23. Goin MK, Rees TD. A prospective study of patients' psychological reactions to rhinoplasty. Ann Plast Surg. 1991; 27 (3): 210-215.

24. Gorney M. Ten years' experience in aesthetic surgery malpractice claims. Aesthet Surg J. 2001; 21 (6): 569571.

25. Rohrich R. Silicone breast implants: outcomes and safety update. Plast Reconstr Surg. 2007; 120 (Suppl 1): 1S-3S

26. McGrath $\mathrm{MH}$. The psychological safety of breast implant surgery. Plast Reconstr Surg. 2007; 120 (7 Suppl 1): 103S-109S.

27. Breiting VB, Hölmich LR, Brandt B, Fryzek JP, Wolthers MS, Kjøller K et al. Long-term health status of Danish women with silicone breast implants. Plast Reconstr Surg. 2004; 114 (1): 217-226; discussion 227-228.

28. de Brito MJ, Nahas FX, Barbosa MV, Dini GM, Kimura $A K$, Farah $A B$, Ferreira LM. Abdominoplasty and its effect on body image, self-esteem, and mental health. Ann Plast Surg. 2010; 65 (1): 5-10.

29. Javo IM, Sørlie T. Psychosocial predictors of an interest in cosmetic surgery among young Norwegian women: a population-based study. Plast Reconstr Surg. 2010; 126 (2): 687-688; author reply 688.

30. Koot VC, Peeters PH, Granath F, Grobbee DE, Nyren O. Total and cause specific mortality among Swedish women with cosmetic breast implants: prospective study. BMJ. 2003; 326 (7388): 527-528.

31. O'Blenes CA, Delbridge CL, Miller BJ, Pantelis A, Morris SF. Prospective study of outcomes after reduction mammaplasty: long-term follow-up. Plast Reconstr Surg. 2006; 117 (2): 351-358.

32. Pukkala E, Kulmala I, Hovi SL, Hemminki E, Keskimäki I, Pakkanen M et al. Causes of death among Finnish 
women with cosmetic breast implants, 1971-2001. Ann Plast Surg. 2003; 51 (4): 339-342; discussion 343-344.

33. Brinton LA, Lubin JH, Murray MC, Colton T, Hoover RN. Mortality rates among augmentation mammoplasty patients: an update. Epidemiology. 2006; 17 (2): 162-169.

34. Villeneuve PJ, Holowaty EJ, Brisson J, Xie L, Ugnat AM, Latulippe L et al. Mortality among Canadian women with cosmetic breast implants. Am J Epidemiol. 2006; 164 (4): 334-341.
Correspondencia:

\section{Dr. André Víctor Baldin}

Hospital General «Dr. Manuel Gea González» $2^{\circ}$ Piso - Cirugía Plástica y Reconstructiva.

Av. Calzada de Tlalpan Núm. 4800,

Col. Sección XVI, 14080, Tlalpan,

Ciudad de México, México.

Teléfono: 4000 3000, ext. 3721

E-mail: andre.baldin03@gmail.com 